\title{
Formas comunicacionales de resistencia indígena campesina frente al Proyecto Minero Kimsakocha en Ecuador
}

\author{
Communication forms of peasant indigenous resistance against \\ the Kimsakocha Mining Project in Ecuador
}

Julio Lizardo Zhagui Pérez ${ }^{1}$ César Leonidas Ruiz ${ }^{2}$

\section{Resumen}

Esta investigación ha analizado el rol de los elementos comunicacionales de resistencia indígena campesina, frente al Proyecto Minero Loma Larga (Kimsakocha), de Ecuador. Es un estudio cualitativo desde una perspectiva vivencial simbólico que se sustenta en cuatro momentos: vivenciando y experienciado; recuperando y reencantando; significado y resignificado; e involucrándonos y comprometiéndonos con una solución compartida. Los principales resultados describen la realidad de la minería en el Ecuador, los procesos llevados a cabo por el Estado para poner en marcha la política económica extractivista por encima del bienestar de las mismas comunidades, así mismo, las entidades que ha creado para la regulación del tema minero y el recuento del proceso de la lucha jurídica y las acciones emprendidas por las comunidades, con énfasis en la participación de la mujer en contra de la extracción minera en Kimsakococha. A manera de conclusión, se confirman que las formas de comunicación tanto ancestrales como modernas son determinantes e indispensables para el desarrollo de la resistencia frente a la vulnerabilidad del territorio indígena.

Palabras clave: Comunicación ancestral; procesos organizativos; resistencia indígena; política económica; extractivismo minero.

\section{Abstract}

This research has analyzed the role of the communicative elements of indigenous peasant resistance, against the Loma Larga Mining Project (Kimsakocha), from Ecuador. It is a qualitative study from a symbolic experiential perspective that is sustained in four moments: living and experiencing; recovering and re-enchanting; meaning and resignified; and getting involved and committing to a shared solution. The main results describe the reality of mining in Ecuador, the processes carried out by the State or Government to implement the mining economic policy above the wellbeing of the

\footnotetext{
1 Máster en Comunicación Intercultural con Enfoque de Género. Periodista de la Confederación de Pueblos de la Nacionalidad Kichwa del Ecuador (ECUARUNARI). Email: lizardozhagui1517@gmail.com

2 Antropólogo e Investigador de la Pluriversidad Amawtay Wasi del Ecuador. Email: intituksiy@hotmail.com
}

Recibido: 26/02/2018 Aprobado: 28/08/2018 
communities themselves, as well as the entities that the government has created for the regulation of mining issue and the recount of the legal struggle process and the actions undertaken by the communities, with emphasis on the women participation against mining extraction in Kimsakococha. As a conclusion, it is confirmed that both ancestral and modern forms of communication are determinant and indispensable for the development of resistance to the vulnerability of the indigenous territory.

Keywords: Ancestral Communication; organization processes; indigenous peasant resistance; economic policy; mining extraction.

\section{Introducción}

El objetivo de esta investigación fue analizar el rol que cumplen las formas comunicacionales presentes en las luchas de la resistencia indígena campesina, frente al proyecto minero Kimsakocha, en Tarqui, Victoria del Portete y Girón Azuay-Ecuador. Existe una preocupación por las comunidades indígenas campesinas desde la llegada de las empresas privadas y estatales que tienen la finalidad de extraer oro, cobre y plata, afectando la vegetación, la hidrografía así como violentando la sagrada Pachamama (Madre Tierra).

Sabiendo que los pueblos originarios desde siempre han convivido con el agua, como uno de los cuatro elementos vitales de la naturaleza que fertiliza sus chakras, buscando desde sus vivencias, la armonía entre ellos, para lo cual han trabajado en comunidad y desde sus organizaciones con el fin de mantener sus fuentes y evitar la contaminación y el daño de las mismas. Es por ello, que la importancia de esta investigación, radica en el aporte que representa para las comunidades indígenas, rescatar y revalorizar las formas ancestrales de comunicación, estudiar los instrumentos que se utilizaban desde siempre para convocar a la comunidad, reunirse y consensuar, lo que a su vez redunda en el rescate de valores culturales ancestrales.

\section{Revisión de literatura}

\section{La comunicación de los pueblos indígenas}

Al hablar de comunicación de los pueblos indígenas nos referimos a la expresión de su visión, demandas y propuestas, de una comunicación que responde a situaciones de exclusión, discriminación y hasta exterminio del sujeto histórico, es decir, los pueblos indígenas que luchan por la conservación de sus identidades en un mundo globalizado. Se define a la comunicación como un "poner en común", pues bien, a lo largo de la historia, los pueblos indígenas han desarrollado un variado y diverso abanico de modos o medios para esa puesta en común. 
Bajo esta perspectiva, coincidimos también con la posición expresada por representantes de los pueblos indígenas frente a la Cumbre Mundial de la Sociedad de la Información (CMSI, 2003): la comunicación es una práctica social cotidiana y milenaria de los pueblos indígenas que es fundamental para la convivencia armónica entre los seres humanos y la naturaleza. Para los pueblos indígenas la comunicación es integral pues parte de una cosmovisión en la cual todos los elementos de la vida y la naturaleza se hayan permanentemente relacionados e incluidos entre sí. Por esta razón la comunicación tiene como fundamento una ética y una espiritualidad en el que los contenidos, los sentimientos y los valores son esenciales.

La comunicación para los pueblos indígenas es inherente a ellos como sujetos sociales y reflejados en sus expresiones y en su identidad. Como lo afirma Almendra (2010), la esencia de la comunicación no está en los medios que se utilizan sino en cómo los transforman y recrean para ponerlos al servicio de la organización, del proceso y de la conciencia colectiva. Los pueblos recrean lo que de afuera les sirve, siempre y cuando responda a las necesidades y al contexto que vive su comunidad.

Por su naturaleza y origen popular, la comunicación indígena posee los rasgos de una comunicación comunitaria y participativa, pues se trata de iniciativas comunicativas que surgen de la comunidad ante la necesidad de visibilizar las demandas de los pueblos indígenas ante la sociedad, en este caso la defensa de su territorio y de sus recursos, con lo cual se fortalecen las organizaciones y se articulan con los diferentes actores de la sociedad para generar cambios.

\section{Medios de comunicación ancestrales}

La comunicación desde la cosmovisión y cultura de los pueblos indígenas, tiene una estrecha relación con su entorno, es decir, se entiende la comunicación en una relación constante con la Pacha Mama o Allpa Mama; esta relación se da a través de los rituales para la cosecha y la siembra - con las concentraciones comunitarias, por el uso de los instrumentos propio de alertas y llamados como es la bocina, el churu (concha), y otros instrumentos (Chela 2003, citada por Chují, 2007).

Aparte de esa relación intrínseca con su entorno, la comunicación en el mundo indígena se la concibe como ese espacio vivo de secuencias que se concretan en la transmisión, intercambio, re-generación de conocimientos ancestrales y actuales, como una 'herencia oral' viva de la sabiduría acumulada de los antepasados. (CMSI, 2003).

Cuando hablamos de medios de comunicación ancestrales nos referimos a los instrumentos, a los métodos que utilizaban en la antigüedad para transmitir mensajes, para convocarse, para informar de la llegada de intrusos, etc., medios de carácter comunitario pues llegaban y provenían de todos los miembros de la comunidad. Ejemplo de esto es el uso de la bocina como instrumento utilizado para convocar a 
las asambleas, a las mingas, o el churo con el que no solo se convocaba, sino que se alertaba de la presencia de personas ajenas al territorio.

\section{Materiales y métodos}

Esta investigación es cualtitativa planteada desde una perspectiva vivencial simbólica que nace de la vivencia y descripción de los hechos por parte de sus protagonistas y cuyos resultados representan insumos importantes para el compromiso con la comunidad con importancia incluso a nivel nacional, que permite un adecuado análisis de la realidad e implica mejoras en los procesos de lucha. Con este fin, se contemplan cuatro momentos: vivenciando y experienciando; recuperando y reencantando; significando y resignificando; involucrándonos; y comprometiéndonos (RUIICAY, 2015, pp. 15-16).

Los participantes en la investigación han sido dirigentes y algunos miembros de las parroquias Tarqui, Victoria del Portete y el Cantón Girón en el Ecuador. A ellos, se les aplicó instrumentos como la entrevista, se utilizó la observación participativa en las comunidades, en el mismo páramo y para finalizar, se hizo análisis de documentos.

\section{Resultados y discusión}

\section{Proyecto Loma Larga-Kimsacocha}

El estudio ha ayudado a caracterizar el proyecto Minero Loma Larga-Kimsakocha: el páramo Kimsakocha, ubicado en la Cordillera Occidental de los Andes, en el bosque de protección y zona de vegetación de la cuenca del río Paute y la micro cuenca de los ríos Yanuncay e Irquis. Está localizado cerca de cuatro parroquias: Victoria de Portete y Tarqui del cantón Cuenca, San Gerardo del cantón Girón, Chumblín del cantón San Fernando, en la provincia del Azuay y afecta a otras comunidades cercanas al proyecto. La mayor parte de la concesión se encuentra dentro del ecosistema y páramo de Kimsacocha, nacen 35 sistemas de agua que abastecen a las poblaciones cercanas. El $87 \%$ del caudal de estos sistemas se destina al consumo humano, actividades agrícolas, riego, piscícola y ganadería. La zona de mineralización de oro principal se centra en las coordenadas geográficas: Latitud: $03^{\circ}$ o3'S Longitud: $79^{\circ} 13^{\prime} \mathrm{W}$.

Se encontró que Kimsacocha fue, hasta el año 2012, parte de un área de páramo concesionada para la explotación minera, pero el Ministerio del Ambiente recuperó 3.217 de las 12.000 hectáreas dadas en concesión y las declaró área nacional de recreación en el sector conocido como Las Tres Lagunas. La importancia ecológica de este volcán y lo cual es el motivo de lucha de las comunidades en cuestión, son sus características hídricas, típicas de arroyos de alta montaña, la alta pluviosidad, resquebrajamiento de las rocas del subsuelo y la regular morfología del terreno que favorecen la acumulación de grandes cantidades de aguas superficiales en forma de lagunas y cauces de lento caudal y aguas infiltradas en el subsuelo. 
Inicialmente el proyecto se denominaba Kimsakocha (Tres Lagunas), bajo la responsabilidad de la compañía Iam Gold, hoy es propietaria la empresa INV Metals, la misma que le cambió de nombre, hoy conocido como Loma Larga, ubicado al suroeste de la ciudad de Cuenca, cubre un área aproximadamente de 8 mil hectáreas y tiene una tasa de procesamiento de mil toneladas de mineral. En este páramo no se registran asentamientos humanos, por ser fuentes hídricas, sin embargo, la propiedad de la tierra se caracteriza por ser comunitaria.

\section{La minería en el Ecuador}

De la revisión documental, se encontró que en Ecuador, el tema minero no tenía una importancia trascendental cuando se hacía de manera artesanal y no causaba mayor impacto ambiental. Sin embargo, las cosas cambiaron con la llegada a la presidencia de Rafael Correa, ya que impulsó la minería a gran escala para que se explotaran los minerales, con el fin de atraer capitales, considerando que Ecuador cuenta con un potencial de reservas de metales como el oro, cobre, y plata, localizados en diferentes zonas del territorio. En cumplimiento de esta política extractivista, en 2015 se crea el Ministerio de Minas, como ente rector y ejecutor de la política minera del área geológica-minera de conformidad con los principios de sostenibilidad, precaución, prevención y eficacia, además es parte del sector estratégico del país.

En la actualidad, existen varias entidades que regulan la actividad minera en el Ecuador que cumplen cada una de ellas una función importante en el control de la actividad. En primera instancia el Ministerio de Recursos Naturales no Renovables, encargado de las políticas públicas del sector, la Agencia de Regulación y Control Minero (ARCOM), encargado del ejercicio de la potestad estatal de vigilancia, auditoria, intervención y control de las fases de la actividad minera; el Instituto Nacional de Investigación Geológico Minero Metalúrgico (INIGEMM), encargado de realizar actividades de investigación, desarrollo tecnológico e innovación en materia geológica, minera y metalúrgica (Art. 10, Ley actual de Minería, 2009), la Empresa Nacional Minera, destinada a la gestión de la actividad para el aprovechamiento sustentable de los recursos, materia de la presente ley en observancia a las disposiciones de la misma y sus reglamentos.

Se ha visto que la tesis del gobierno para la promoción y el apoyo a la minería a gran escala estaba en el hecho de que ésta iba a traer un sin número de beneficios para las poblaciones cercanas a los proyectos, tales como creación de escuelas, hospitales, carreteras, trabajos, hidroeléctricas, etc., y ha optado por entregar las concesiones a transnacionales mineras. En la actualidad en el territorio ecuatoriano, hay un total de un mil treinta y seis propietarios mineros, inscritos y otorgados; y 2 mil 257 concesiones inscritas, otorgadas y en trámite, que abarcan 1, 21 millones de hectáreas, es decir el 4,5\% de la superficie total del país. 


\section{Los pueblos en resistencia y la lucha jurídica}

Se logró evidenciar que la lucha de las comunidades indígenas y campesinas no se ha limitado a movilizaciones y cierre de carreteras, al contrario, también se ha llevado a cabo una lucha jurídica para asegurar los derechos que se han ganado a lo largo de los años, cuando se habla de derechos colectivos y de la naturaleza, es hablar, de los derechos adquiridos desde el Convenio 169 (OIT, 2007), en lo referente a la obligatoriedad de la consulta previa, el derecho de propiedad, el derecho a participar en la utilización, administración y conservación de dichos recursos.

Se ha podido ver en estos procesos, que el gobierno ha pasado por alto los derechos adquiridos, negando el derecho a la consulta previa e informada, imponiendo bien sea a la fuerza o convenciendo a las personas de beneficios que nunca llegarían y que están por encima de su cultura, de sus necesidades de vida y comunión con la Pacha Mama, prometiendo obras a partir de las regalías, como escuelas, hospitales, carreteras, etc., desconociendo lo que es vital para las poblaciones que es la vivencia con su entorno a partir y alrededor del páramo, no teniendo en cuenta que para las poblaciones indígenas es más importante el encuentro y la vida armoniosa con el agua, con las plantas, con la agricultura, su sustento, planteándoles un cambio de vida a cambio de vivir en una selva de cemento.

Por otro lado, se han querido violentar los derechos de la naturaleza estipulados en la constitución política de 2008, que menciona la restricción de actividades que puedan conducir a la extinción de especies, la destrucción de ecosistemas o la alteración permanente de los ciclos naturales y el derecho a beneficiarse del ambiente y de las riquezas naturales que les permitan el buen vivir. Además de lo escrito en la Ley de Aguas, promulgada en 2013, que también habla de la consulta previa e informada de todas las acciones que afecten la gestión de los recursos hídricos, igualmente el derecho a participar en el uso, usufructo y gestión comunitaria del agua que fluya de sus tierras y territorios, permitiéndose su participación en la planificación y gestión del recurso.

Otro de los derechos de los pueblos, violentados por el gobierno y evidente en la Ley de Comunicación promulgada en 2013, es el derecho a ser informados y difundir sus pensamientos, pues no se ha informado de manera adecuada y oportuna sobre lo que realmente implica la puesta en marcha del proyecto minero, ocultando información, prometiendo beneficios que a la larga no llegarían sino a manos de las empresas trasnacionales y a partir de este desconocimiento, algunas autoridades han llegado a firmar convenios interinstitucionales con los dueños de la concesión minera, en las parroquias objeto de esta investigación, aún a espaldas de las mismas comunidades.

Se logró constatar que lamentablemente el Gobierno de Ecuador, a través de enmiendas a la Constitución Política ha limitado la independencia de la justicia y ha 
adicionado artículos que criminalizan la protesta social, obstaculizando las acciones en contra de los proyectos mineros, a pesar de lo cual, las comunidades a través de sus organizaciones permanecen en pie de lucha por sus derechos y los derechos de la naturaleza. Derechos que igualmente se han visto amenazados por el modelo económico del gobierno, que se basa en la acumulación de capital a través de la extracción de recursos naturales, afianzado a través del Plan Nacional de Desarrollo Minero de 2011, que atenta contra los derechos de los pueblos asentados en los territorios donde se encuentran los recursos.

Frente a la política extractivista de recursos naturales petroleros y mineros, los pueblos indígenas y campesinos han llevado adelante, procesos de defensa y resistencia, a los cuales se les ha aplicado la criminalización, reportándose más de 200 personas enjuiciados o investigadas. La causa principal es aplicar el derecho a la resistencia. En todo este proceso de lucha es importante resaltar el trabajo y la lucha emprendida a través de la gestión comunitaria del agua, a través de la Federación de Organizaciones Campesinas e Indígenas del Azuay (FOA), la cual interviene activamente en los debates nacionales sobre el manejo del agua, rechazando algunas propuestas de la reforma a la política pública generada en el gobierno militar de 1970, el mismo que contemplaba la privatización del agua, dejando sin efecto la gestión directa del líquido vital por parte de los usuarios organizados.

Se ha encontrado que la lucha que llevan a cabo diferentes organizaciones indígenas-campesinas, sistemas comunitarios de agua para consumo humano y juntas regantes en la provincia del Azuay, se han dado a conocer a nivel nacional, conformando grupos de trabajo para sacar adelante todo este proyecto de lucha y oposición, se ha generado apoyo para la lucha en las calles, sabiéndose defender de los cargos penales (explotación minera en el páramo de Kimsacocha, Macías Mario).

De las entrevistas realizadas, se destaca lo dicho por los lideres indígenas por la defensa del agua, Carlos Pérez Guartambel, Presidente de la Ecuarunari y del Sistema Comunitario de Agua de las Parroquias Tarqui y Victoria del Portete y por el señor Rigoberto Sánchez, Presidente de los Sistemas de Riego del Cantón Girón, quienes dan a conocer detalles de los inicios de la lucha, indicando que es a partir del 2001 que se inicia la lucha en contra de los proyectos mineros que afectan el territorio de los pueblos indígenas, cuando las comunidades se enteraron que había una compañía haciendo estudios para extraer minerales, denominada Iam Gold, en el momento de enterarse no tenían un plan de acciones trazado, se iniciaron reuniones para analizar la situación y tomar decisiones. En estas circunstancias, empezó un proceso de resistencia fuerte, aunque de pocos, y con autoridades, instituciones educativas y medios de comunicación en contra. Sin embargo, se llevó a cabo la lucha, mediante movilizaciones, levantamientos, cierres de carreteras, superando la criminalización, detenciones, encarcelamiento, y se logró detener la arremetida. Es de resaltar la fuerza que pusieron especialmente las mujeres, quienes con su resistencia lograron que el 
pueblo, no solo sus comunidades sino la ciudad, hicieran conciencia de la afectación que se venía para su territorio y su modo de vida. Al hablar de la mujer defensora del agua de Kimsakocha, se habla de la abuelita, madre, hija, nieta, la agricultora, ganadera, quehaceres de casa, la estudiante, intelectual, maestra, doméstica, comerciante, bordadora, sastre, viajera, que día a día se esmera por el bienestar de los suyos, quienes con su sabiduría investigaron el significado de las palabras "extractivismo y pobres sentados sobre un saco de oro", palabras del primer mandatario que se grabaron en cada una de las mentes de las mujeres luchadoras. Quienes tuvieron la oportunidad se prepararon, asistieron a talleres, asambleas, con el fin de tener conocimiento del tema y poder luchar con conocimiento de causa y hacer conciencia en sus comunidades.

En la actualidad existe un pedido de consulta popular en la corte constitucional, sobre el tema minero realizado por dirigentes y moradores del cantón Girón, quienes proponen que no sea una persona o el estado quien decida el destino de ese pueblo, sino que sean sus habitantes y en las urnas, a través de un proceso transparente libre, voluntario y bien informado y esa es la consulta popular bajo la pregunta: ¿Está usted de acuerdo que se den actividades mineras en el sistema hidrológico de Kimsakocha? Si o No, eso tiene toda legitimidad, la pregunta es diáfana, transparente y constitucional. (Entrevista, líder Indígena, 2016).

\section{Formas de comunicación utilizadas en la resistencia en contra de la minería a gran escala en Kimsakocha}

Durante toda su existencia, los pueblos originarios han tenido formas de comunicación propias, su mismo modo de vida, su visión de la naturaleza, de su misma existencia, del tiempo, del espacio, su cosmovisión, sus estilos propios de vida, comunican con su voz, con su caminar, con todo su ser, y esa comunicación no es solo entre miembros de la comunidad, también se comunican con todo lo que es parte de ellos, porque para los pobladores, los seres humanos no son los dueños y amos de la naturaleza, solo son parte, igual que el agua, que las piedras, los árboles, los animales; para los pueblos, todos los seres de la naturaleza tienen vida y por lo tanto son sujetos de comunicación.

Para comunicarse, desde antes de la llegada de los españoles, los pueblos indígenas utilizaron los instrumentos que les ha proporcionado la naturaleza o que han hecho con objetos de la naturaleza, para llamar, para convocar las asambleas, las mingas, las fiestas. A continuación se destacan la formas de comunicación ancestral y las formas de comunicación modernas.

\section{Formas de comunicación ancestrales}

La bocina comunitaria: instrumento ancestral, que fue utilizado en las fiestas de los pueblos sobre todo en las labores agrícolas, para el pastoreo, para dar alarma por si aparecían enemigos o gente ajena a las tierras, también era visto como sagrado 
para los "yachas" (sabios), quienes aducen que, con su sonido, se concentraban las energías en los rituales. Este instrumento aún sigue siendo en muchas comunidades un instrumento de comunicación efectivo, ya que basta subir a un lugar elevado y entonarla, su sonar recorre el viento para que todas las personas de las comunidades sepan que algo sucede, que para algo se está llamando, convocando, es una de las mejores formas de convocatoria.

Anteriormente era construida con cuernos de vaca y de toro, pero no cualquiera, solo los abuelos sabían escoger los indicados, en la actualidad, es construida por diferentes materiales de preferencia caña de bambú, los espinos de la penca, caña guadua y el cuero de borrego. Antiguamente, la bocina era utilizada como un medio de comunicación entre los indígenas, hoy nuevamente rescatada en las minkas, aglomera a las personas a un lugar seleccionado para los trabajos programados, ha sido utilizada en las convocatorias y marchas en defensa del agua durante los recorridos en contra del extractivismo.

La quipa o churo: la quipa, churo o caracol marino es un instrumento aerófono ancestral que era utilizado hace miles de años para mensajería. Es un caracol marino perforado en uno de sus extremos, por el cual se sopla para producir sonidos. Se ha registrado como originario de las culturas Las Vegas, Valdivia y Chorrera, gracias a evidencias arqueológicas. En la antigüedad, el uso de este instrumento era exclusivo de los chasquis o mensajeros. En la actualidad es utilizado por los pobladores para convocar a trabajos comunitarios en mingas o para llamar a estar presente en acontecimientos de mucha importancia, como las fiestas ancestrales, avisos, señales, asambleas, la cosecha y en nuestro caso para el llamado a la resistencia.

Gracias a sus características tímbricas y sonoras, el caracol marino puede ser escuchado a largas distancias, más aún si sus ejecutantes se ubican en zonas altas como montañas o mesetas. Es por ello, que su timbre grave y melancólico era utilizado para pedir permiso a los apus, montañas consideradas divinidades, para realizar cualquier actividad. Las distintas sonoridades que la quipa producía causaban una reacción en la gente de la comunidad, quienes decodifican el mensaje y actuaban de acuerdo a un significado social preestablecido.

El cohete artesanal: se emplea en exhibiciones, festejos, festividades, celebraciones, etc. En esta ocasión son utilizados cuando existe la presencia de personas ajenas a la comunidad y que desean socializar las bondades de la minería. Su sonido pone en alerta a la vecindad, que rápidamente se enteran de lo que está ocurriendo.

El grito: Es una vocalización ruidosa, en el campo, los vecinos aplican constantemente para informar cualquier antecedente, es común escuchar en las zonas rurales el grito de aviso. Es de destacar en este proceso de defensa del Páramo que dotaba de agua a las comunidades, estas formas propias de comunicación, en el sentido de que 
las mismas formas organizativas son de carácter vertical, en las que las convocatorias a asambleas, marchas, etc., tienen carácter obligatorio, por ejemplo, si la junta de agua hace el llamado a las comunidades para participar en una marcha en contra del proyecto, o si convocan a asamblea y se toma la decisión de no dejar las tierras, son decisiones que no se discuten y se tienen que cumplir, y los pobladores en ningún momento sienten que se les esté obligando a hacer algo que no quieren hacer porque ellos aceptan estar en una organización, confían en las decisiones y formas organizativas de sus dirigentes y por lo tanto, cumplen lo que se dice, sin discusión.

\section{Formas de comunicación actual}

Los altos parlantes ubicados en las casas comunales: es un medio utilizado para generar avisos y convocatorias, con éste se evita visitar los hogares pues es un medio que se expande el sonido en un amplio radio de acción, se adecúan en lugares estratégicos para que sean escuchados por todos y así ahorrar tiempo.

Los parlantes movibles: son traductores electroacústicas utilizados para las convocatorias y avisos, que generalmente van ubicados en vehículo o llevados a mano, y que invitan a las minkas o asambleas de cualquier índole.

Las banderas como símbolos: en esta lucha anti minera han servido como símbolo de resistencia, llevan diferentes logotipos y mensajes, son colocadas en los balcones y ventanas de las viviendas, son portadas para las marchas y concentraciones. Se le ubica en este apartado porque no solo es un símbolo, la presencia de la bandera comunica un acontecimiento especial, una convocatoria, es un llamado a la comunidad a reunirse en torno a algo importante.

Las campanas de las iglesias comunitarias: un dispositivo simple que emite un sonido. Sirven como emergencia y puesta en alerta. Su ejecución es diferente a lo normal. A las cuerdas se les aplican más de prisa cuando se trata de algo urgente.

El periódico mural: estos son utilizados especialmente en las casas comunales, donde se puede encontrar gran cantidad de información de los sucesos que ocurren en los pueblos, por lo general son responsables de lo que se publica los presidentes de las comunidades.

La fotografía: aprovechando las festividades y eventos en las comunidades, se realizan exhibiciones fotográficas, con temas relacionadas a la lucha por defender el agua, se hace un recuento de las fotos que se realizaron en los primeros años de resistencia, así como las realizadas en las últimas fechas.

Videos: gracias a un grupo de jóvenes entusiastas y aficionados a la filmación, se han editado algunos videos, especialmente relacionados a la lucha anti minera, los 
mismos que han sido rodados en diferentes escenarios e incluso han servido para compartir dentro y fuera del país, con los testimonios desarrollados en esta zona. Por ejemplo, la consulta comunitaria realizada por los dirigentes de Tarqui y Victoria del Portete, con la finalidad de preguntar a la gente si aceptan o no la explotación minera en Kimsakocha.

Hojas volantes: son utilizadas con convocatorias o información de las actividades que realizan los dirigentes, ya sea en la ciudad o fuera de ellas, también sirven para sacar resúmenes de leyes y acuerdos ministeriales, especialmente del agua, minería y medio ambiente.

La radio: siendo un medio de sintonía masiva y aprovechando que es utilizado por todos los estratos sociales, se han utilizado ya sea por medio de contrato y por cortesía de sus dueños, no así los manejados por el gobierno, que han cerrado las puertas. Este medio en gran parte ha sido aprovechado para que los dirigentes puedan participar en debates y aclaraciones cuando el oficialismo ha arremetido con sus argumentos a favor de la minería. Lo importante de este medio especialmente en Cuenca-Azuay, los ejecutivos son personas progresistas y valoran el agua y la lucha campesina, en varias acciones también han participado emisoras regionales y nacionales.

El periódico comunitario: un grupo de jóvenes aficionados en lo referente a la comunicación alternativa, con el apoyo de dos profesionales periodistas, fundaron un periódico comunitario de nombre SENDERO, medio alternativo que jugó un papel muy importante en la resistencia anti minera. El medio al inicio circulaba cada mes, pero por problemas económicos se tuvo que alargar la circulación a tres meses. Siempre se estuvo cubriendo información a la situación de la lucha de los defensores del agua, porque era en medio netamente comunitario e indígena. Sus directivos tuvieron que enfrentar dos juicios instaurados por autoridades que se sentían ofendidos, pero más pudo la verdad, los procesos se archivaron porque los jueces no encontraron nada de culpabilidad, al contrario, las acciones fueron por tratar de suspender su presencia, lamentablemente se tuvo que cerrar definitivamente por problemas de índole laboral y económico, luego de cuatro años de circulación.

Los periódicos que circulan en la región y ciudad: estos son dos, Diario El Mercurio y El Tiempo, de propiedad privada, que han cubierto todos los acontecimientos desarrollados hasta la fecha.

Periódico Ají: es un medio alternativo del GAD Provincial, circula cada mes, difunde noticias de las comunidades, también cubren información sobre temas del medio ambiente y en este caso los problemas mineros en la provincia.

Canales de TV locales: existen tres canales regionales, siendo estos, Tele Cuenca canal universitario católico, que en gran parte han cubierto los acontecimientos, 
sobre todo cuando se han dado marchas hacia la Ciudad de Cuenca. Unsión TV, canal de programas blancos como ellos lo denominan, cuando se han tratado de acciones fuertes de los protestantes siempre están presentes. Telerama, canal perteneciente a una familia económicamente muy poderosa como es los Eljuri, así mismo cuando se trata de movilizaciones masivas sus periodistas se hacen presentes, alguna vez llegaron hacia el páramo.

Canales nacionales: tiene su equipo de reporteros en Cuenca, gran parte de los acontecimientos han difundido, también elaboraron reportajes del Kimsakocha. Tele Amazonas, es un poco más prudente al difundir la noticia, por el miedo que les tienen controlado los órganos del estado, ellos prefieren ignorar muchas cosas. Ecuavisa canal nacional, que ha acompañado en todas las acciones anti mineras desarrolladas por los campesinos. Ellos se encuentran sigilosamente vigiladas por las autoridades de control de telecomunicaciones.

Radios on-line: poco a poco la gente va inaugurando este tipo de radios, se ve especialmente los ambientalistas quienes llevan la batuta en estos medios, así como las organizaciones e instituciones con el fin de difundir todo tipo de programas. Por ejemplo, la Federación de Organizaciones Campesinas e Indígenas del Azuay FOA, hace un año fundó la Radio Online, que lleva el nombre Radio Online Kimsakocha, allí colaboran jóvenes comprometidos en la defensa de la vida y el medio ambiente, siempre realizan programas anti mineros, difunden materiales muy seleccionados para el conocimiento de los oyentes, medio que tiene hasta el momento una alta sintonía, el trámite legal de funcionamiento se encuentra en camino. Caben mencionar que, desde que se impuso la nueva Ley de Comunicación en el país, el periodismo Cuencano es muy unido se reveló ante el gobierno, hay mucha solidaridad entre los comunicadores, es decir siempre se está cuestionando las imposiciones ordenadas por el régimen, en definitiva, puede ser un motivo especial para que todos los medios se hayan unido a la gran causa de defender el agua.

Redes sociales: hoy en día con el avance de la tecnología, estos símbolos o instrumentos de comunicación desempeñan un rol importante en la comunicación, se debe preguntar quién no tiene un celular, salvo que no existan antenas colocadas en los sectores campesinos, al menos en los lugares donde se pretende realizar actividades extractivitas, hay servicio de las operadoras, lo mismo ocurre con el internet, que es más manejada por la juventud. Al momento todo tipo de convocatoria es realizada a través de estos medios.

\section{Conclusiones}

La investigación realizada nos lleva a concluir que el Estado del Ecuador, ha utilizado múltiples estrategias para imponer su política extractivista con el fin de aumentar los recursos económicos, sin tener en cuenta las necesidades y la vida misma de los 
pueblos que viven de los recursos hídricos que provienen del Páramo de Kimsacocha, ni la afectación misma a la naturaleza; por un lado, ha hecho modificaciones en la constitución con el fin de favorecer su política minera, criminalizar la protesta, intervenir en la justicia con el fin de doblegar a las comunidades para que acepten el proyecto minero. Por otro lado, ha utilizado el mecanismo de dividir a las comunidades, cooptando y comprando voluntades en las instancias de gobierno nacional y local para lograr establecer los proyectos mineros.

Otra de las estrategias del Estado para convencer a las comunidades de las bondades del proyecto minero, es precisamente la estrategia comunicacional de censura a la información, utilizando los aparatos del estado para coartar la libertad de expresión, amparado en la Ley Orgánica de Comunicaciones expedida en 2013, la cual impulsa la recalificación de los medios de comunicación con el fin de volverlos eficaces y profesionalizarlos para que informen de manera ética.

En este momento todos los medios de comunicación están en ese proceso de recalificación y no pueden formar ni informar libremente a la población, hacia un adecuado criterio ciudadano respecto a la defensa de la naturaleza, a impulsar el valor de los recursos naturales, la importancia del agua, de los páramos para la vida de los pueblos que viven de sus recursos hídricos y del planeta entero que respira a través de su vegetación. De otro lado, utiliza los medios de comunicación estatales (TV Ecuador, TC Televisión, Televicentro, Gama TV, Canal 42 y algunas radios) para impulsar las obras de infraestructura que se han construido con las regalías de la explotación minera, dando a la población la falsa idea de que son más importantes las obras que la Pachamama (Madre Tierra), los recursos que provee, el aire, los modos de vida de las comunidades que viven de esos recursos. Es así como a través de todo este despliegue propagandístico, ha logrado convencer a muchas personas de la bondad de su política minera.

Lo positivo de lo encontrado en la presente investigación es que, a pesar de todos los esfuerzos hechos por el gobierno a través de su aparato estatal, de los mecanismos judiciales, estrategias comunicacionales, es la fuerza organizativa de las comunidades la que ha triunfado, aún a costa de la criminalización de su protesta, bajo la cual se ha enjuiciado y encarcelado a muchas personas que se han atrevido a oponerse, a luchar, a resistir en las calles, en asambleas y desde el mismo páramo. Resistencia para cual se han servido no solo de medios de comunicación modernos como los canales de televisión, el internet y algunas radios, sino que, y lo más importante que queremos destacar en el presente artículo, es el rescate de los instrumentos de comunicación utilizados por los pueblos indígenas desde la antigüedad, como la bocina y el churo que han utilizado para convocar a las marchas, asambleas y movilizaciones y que han hecho que finalmente, triunfe la fuerza organizativa, los valores ancestrales indígenas y campesinos y así se conserven los recursos del páramo y la vida de las comunidades. 


\section{GÉNERO E INTERCULTURALIDAD}

\section{Lista de referencias}

Almendra, V. (2010): "Encontrar la palabra perfecta: experiencia del Tejido de Comunicación del Pueblo Nasa en Colombia", Universidad Autónoma de Occidente, Cali, Colombia.

Chuji, M. (2007). Los medios de comunicación indígenas al servicio de los DD.HHy Colectivos. Recuperado en: https://www.alainet.org/pt/node/119937

CMSI (2003). Declaración de los Pueblos Indígenas ante la Cumbre Mundial de la Sociedad de la Información (CMSI), Ginebra.

RUIICAY (2015). Cultivo y Crianza de Sabidurías y Conocimientos - CCRISAC. URACCAN: Managua.

OIT (2007). Convenio 169 de la Organización Internacional del Trabajo. Recuperado en: http://www.oit.org.pe/WDMS/bib/publ/libros/convenio_169_07.pdf. 